\title{
Protocolo de avaliação psicológica para pacientes oncológicos: Uma proposta
}

\author{
Psychological evaluation protocol for cancer patients: A proposal
}

Josafá Lima Ramos ${ }^{[a]}$, Rodrigo Sanches Peres ${ }^{[b]}$

\footnotetext{
${ }^{[a]}$ Especialista em Psicopatologia Clínica pela Universidade Cruzeiro do Sul, psicólogo da Fundação Hospitalar de Hematologia e Hemoterapia do Amazonas, Manaus, AM - Brasil, e-mail: josaramos@hotmail.com

${ }^{[b]}$ Doutor em Psicologia pela Universidade de São Paulo (USP), professor adjunto da Universidade Federal de Uberlândia (UFU), Uberlândia, MG - Brasil, e-mail: rodrigosanchesperes@yahoo.com.br
}

Recebido: 30/01/2012 Received: 01/30/2012

Aprovado: 02/05/2012 Approved: 05/02/2012

\section{Resumo}

Os protocolos de avaliação psicológica se destacam como um recurso potencialmente proveitoso para a prática em Psicologia Hospitalar, na medida em que podem auxiliar na obtenção dos elementos necessários para o planejamento e o desenvolvimento de intervenções. Entretanto, ainda são escassas as publicações brasileiras dedicadas ao assunto. Partindo de tais premissas, este estudo tem como objetivo básico apresentar a proposta de um protocolo de avaliação voltado especificamente a pacientes oncológicos adultos. Tal protocolo se apoia, por um lado, na experiência profissional dos autores e, por outro lado, em uma revisão da literatura científica nacional. Os testes psicológicos que constituem o protocolo são os seguintes: Inventário Fatorial de Personalidade (IFP), Questionário de Saúde Geral (QSG), Inventário de Sintomas de Stress de Lipp (ISSL) e Inventário de Percepção de Suporte Familiar (IPSF). Cada um deles tem discutidas suas características técnicas e indicações frente às particularidades dessa população. Contudo, a aplicação de testes psicológicos é recomendada somente em um momento posterior à obtenção de informações preliminares por meio de anamnese e exame de funções psíquicas e à observação de aspectos relevantes dos pacientes. Os alcances e limites do protocolo são ponderados, mas sua aplicação é recomendada tanto na pesquisa quanto na prática em Psicologia Hospitalar e, mais especificamente, em Psico-oncologia. Considera-se que o mesmo fornece subsídios iniciais para o preenchimento de uma importante lacuna nessas áreas, na medida em que pode viabilizar o acesso a informações essenciais para a assistência integral aos pacientes oncológicos.

Palavras-chave: Testes psicológicos. Planejamento de assistência ao paciente. Administração de cuidados ao paciente. Serviço hospitalar de Oncologia.

\section{Abstract}

Psychological assessment protocols stand out as a potentially useful resource for psychology practice in hospitals, since they can help obtaining necessary information for planning and developing interventions. However, Brazilian publications dedicated to this theme are still scarce. Based on these premises, this study aims to present the proposal of a psychological assessment protocol designed specifically for adult cancer patients. This protocol is based on the authors' professional experience and a Brazilian scientific literature review. The psychological tests that constitute the 
protocol are: Factorial Personality Inventory (IFP), General Health Questionnaire (QSG), Lipp's Symptoms of Stress Inventory (ISSL) and Perception of Family Support Inventory (IPFS). Their technical characteristics and indications in face of this population's particularities are discussed. However, the application of psychological tests is recommended only after the achievement of preliminary information through anamnesis, mental functions evaluation, and observation of relevant aspects from the patients. The scope and limits of the protocol are discussed, but its application is recommended in psychological researches and practices in hospitals and, more specifically, in Psycho-oncology. The protocol provides initial elements to fill an important lack in these areas, since it can facilitate the access to essential information for cancer patients' assistance.

Keywords: Psychological tests. Patient care planning. Patient care management. Oncology service hospital.

\section{Introdução}

A Psico-oncologia se destaca como uma especialidade que se articula dialeticamente entre duas disciplinas científicas: a Psicologia e a Oncologia. Tal especialidade começou a se desenvolver na década de 1970 nos Estados Unidos da América, a partir do momento em que a comunidade científica passou a reconhecer que tanto o surgimento quanto a manutenção e a remissão dos diversos tipos de câncer são intermediados por uma série de fatores que extrapolam as condições de natureza biomédica (Veit \& Carvalho, 2008).

Segundo Holland (2002), a Psico-oncologia tem contribuído de modo significativo para a promoção da qualidade de vida de pacientes oncológicos. Ocorre que, ainda segundo a referida autora, tal especialidade tem como uma de suas preocupações principais a sistematização de um corpo de conhecimentos capaz de fornecer subsídios à assistência integral aos pacientes oncológicos e a suas famílias, por um lado, e, por outro lado, à formação de profissionais de saúde envolvidos com a prevenção, o tratamento e a reabilitação dos diversos tipos de câncer.

A relevância da Psico-oncologia se torna patente levando-se em consideração que o câncer tende a afetar a vida dos pacientes como um todo, sendo, inclusive, muitas vezes vivenciado como sinônimo de sofrimento e até mesmo de morte (Barbosa, Santos, Amaral, Gonçalves, \& Bruscato, 2004). Do ponto de vista físico, os pacientes comumente se sentem fragilizados devido aos sintomas inerentes à doença ou aos efeitos colaterais do tratamento quimioterápico e radioterápico, conforme Carvalho (2002). Do ponto de vista social, o sofrimento decorre, ainda segundo a referida autora, essencialmente do distanciamento dos amigos e da família, da alteração de papéis e da perda da autonomia.

Já do ponto de vista psicológico, os pacientes oncológicos tipicamente apresentam um comprometimento devido ao contato com a própria finitude que se impõe a partir do adoecimento. Afinal, o câncer, para Chiattone (1996), mostra ao sujeito que sua existência está ameaçada e elimina a sensação inconsciente de onipotência frente à morte que caracteriza os seres humanos. Desse modo, muitas vezes desencadeia sintomas de estresse, ansiedade e depressão, contribuindo para a acentuação de conflitos psicológicos prévios, para a eclosão de transtornos psiquiátricos e até mesmo para o surgimento de pensamentos suicidas (Graner, Cezar, \& Teng, 2008).

A assistência integral aos pacientes oncológicos, tal como preconizada pela Psico-oncologia, possibilita que as atitudes e comportamentos dos mesmos em relação à própria saúde venham a ser repensadas. Torna-se possível, assim, auxiliá-los a perceber a necessidade de uma reorganização capaz de contribuir para a adaptação à nova realidade implementada pela doença. Porém, muitas vezes o psicólogo que opta por se dedicar à Psico-oncologia encontra dificuldades para desenvolver intervenções de modo a atingir os objetivos mencionados.

Isso ocorre essencialmente quando o psicólogo não dispõe dos elementos necessários para o planejamento de intervenções. A obtenção desses elementos, por sua vez, pode ser considerada mais complexa para o psicólogo hospitalar. Em parte, esse fenômeno pode ser compreendido levando-se em consideração que, conforme Ribeiro (2005), existem diferenças importantes entre o trabalho do psicólogo em um hospital e em um consultório, local de atuação mais tradicional para esse profissional. 
Ainda de acordo com a referida autora, essas diferenças se organizam em três eixos distintos: o sujeito, o setting e a presença da morte.

No que tange ao primeiro eixo, deve-se esclarecer que o sujeito hospitalizado geralmente apresenta questões orgânicas como a principal temática do sofrimento, o que não é comum em um sujeito que procura um consultório psicológico. Já em relação ao segundo eixo, vale mencionar que o setting é distinto essencialmente porque o trabalho do psicólogo hospitalar é realizado, muitas vezes, em um ambiente de privacidade comprometida e tem início a partir de encaminhamentos, e não de uma demanda espontânea. Por fim, a propósito do terceiro eixo, é preciso enfatizar que, no hospital, a presença da morte é concreta, em contraste com o que ocorre em um consultório psicológico (Ribeiro, 2005).

Além disso, o trabalho do psicólogo hospitalar é bastante complexo, na medida em que, para Simonetti (2004), pode ter como finalidades básicas: a) ajudar o paciente a transitar melhor pelo adoecimento; b) possibilitar o esclarecimento de seus aspectos emocionais principais e/ou c) favorecer seu engajamento no próprio tratamento. Talvez por conta dessa complexidade, muitas vezes o próprio psicólogo hospitalar não consegue ter clareza acerca de suas atribuições, situação essa que, como alertam Bruscato e Benedetti (2004), muitas vezes levam a improvisações que, embora bem-intencionadas, se mostram pouco resolutivas.

Diante do exposto, conclui-se que, para a obtenção dos elementos necessários para o planejamento de intervenções a serem desenvolvidas junto a pacientes oncológicos, o psicólogo hospitalar pode se beneficiar de protocolos de avaliação desenvolvidos especialmente para essa finalidade. Afinal, os protocolos de avaliação são capazes de fornecer parâmetros para a elaboração de um plano de trabalho adequado a partir da sistematização de informações relevantes em termos das particularidades da população assistida pelo psicólogo hospitalar (Capitão, Scortegagna, \& Baptista, 2005).

O emprego de protocolos de avaliação apresenta outra vantagem importante tendo-se em vista que, conforme Lopes e Amorim (2004), o trabalho do psicólogo hospitalar geralmente alcança maior reconhecimento da parte dos demais profissionais de saúde quando se apóia em instrumentos sistemáticos e parâmetros definidos criteriosamente. Entretanto, na literatura científica nacional são escassas as publicações que se dedicam à proposição de protocolos de avaliação passíveis de utilização em Psicologia Hospitalar ou, mais especificamente, em Psico-oncologia. O estudo de Azevêdo (2010), voltado a crianças queimadas, representa uma exceção nesse sentido.

A reversão dessa situação, portanto, pode ser considerada fundamental para a consolidação científica tanto da Psicologia Hospitalar quanto da Psicooncologia no país. Partindo de tal premissa, este estudo tem como objetivo básico apresentar um protocolo de avaliação voltado a pacientes oncológicos adultos. Para tanto, serão caracterizados alguns testes psicológicos capazes de contribuir para a compreensão de diferentes aspectos emocionais importantes para o planejamento e o desenvolvimento de intervenções junto a essa população. Esse recorte se justifica tendo-se em vista que os testes psicológicos - enquanto medidas objetivas e padronizadas de amostras de variáveis psicológicas em suas diversas formas de expressão (Hogan, 2006) - ocupam um lugar central nos protocolos de avaliação.

\section{Proposta de protocolo de avaliação psicológica para pacientes oncológicos}

0 protocolo de avaliação ora proposto se apoia, por um lado, na experiência profissional dos autores em Psicologia Hospitalar e, mais especificamente, em Psico-oncologia e, por outro lado, em uma revisão da literatura nacional realizada a partir de consultas a artigos publicados em periódicos científicos especializados, dissertações, teses, livros e capítulos de livros. Além disso, tem como ponto de partida principal a assertiva de Almeida (1995), a qual estabelece que a escolha dos testes psicológicos a serem utilizados na avaliação de pacientes hospitalizados deve levar em consideração fatores de ordem prática.

Pode-se considerar como um fator de ordem prática a condição dos testes psicológicos em termos de suas propriedades psicométricas. Ocorre que, de acordo com a legislação vigente no país (Resolução CFP n. 02/2003, 2003), somente podem ser empregados no contexto da prática profissional em Psicologia testes psicológicos dotados de indicadores de validade, fidedignidade e precisão cuja adequação é comprovada mediante parecer favorável no Sistema de Avaliação de Testes Psicológicos 
(SATEPSI). Também representa um fator de ordem prática a exequibilidade dos testes psicológicos face às características do ambiente hospitalar, o qual, para Capitão et al. (2005), muitas vezes é desfavorável nesse sentido.

Vale destacar que o protocolo ora proposto, a exemplo daquele elaborado por Azevêdo (2010), envolve a aplicação de testes psicológicos somente em um momento posterior à obtenção de informações preliminares e à observação de aspectos relevantes dos pacientes, como se vê na Figura 1. A obtenção de informações preliminares envolve basicamente o levantamento de dados psicossociais por meio de um roteiro de anamnese nos moldes daquele formulado por Angerami-Camon e Chiattone (2003) e o exame de funções psíquicas tal como preconiza Dalgalarrondo (2000). Já a observação de aspectos relevantes deve contemplar, em linhas gerais, o relacionamento dos pacientes com seus familiares e com a equipe de profissionais de saúde responsável pelo mesmo, de modo a viabilizar, mais especificamente, a compreensão de seus recursos adaptativos.

A aplicação dos testes psicológicos deve ser realizada em conformidade com os achados oriundos desses procedimentos anteriores, uma vez que os mesmos certamente auxiliarão na definição dos objetivos a serem atingidos por meio do emprego do protocolo de avaliação. Feita essa ressalva, deve-se esclarecer que os testes psicológicos que constituem o protocolo ora proposto são os seguintes: Inventário Fatorial de Personalidade (IFP), Questionário de Saúde Geral (QSG), Inventário de Sintomas de Stress de Lipp (ISSL) e Inventário de Percepção do Suporte Familiar (IPSF). Todos eles,

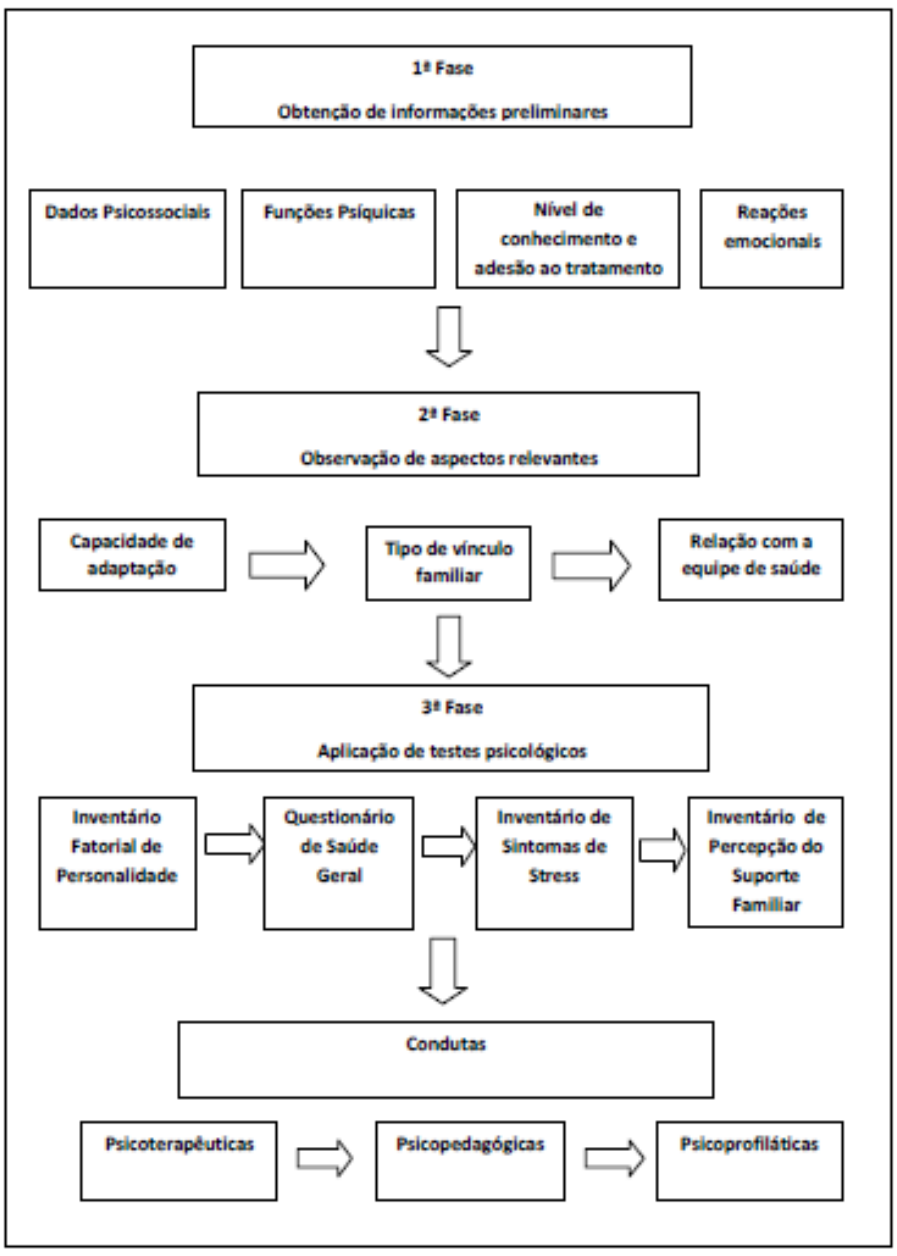

Figura 1 - Protocolo de assistência psicológica a pacientes oncológicos

Fonte: Dados da pesquisa. 
vale destacar, contam com parecer favorável no SATEPSI, de modo que se encontram em condições apropriadas para utilização em termos de suas propriedades psicométricas.

O IFP é um teste psicológico que, como seu próprio nome indica, tem como objetivo a investigação de dimensões da personalidade. 0 instrumento em questão é composto por 155 itens, sendo que 135 deles são relativos a 15 subescalas que avaliam as seguintes dimensões da personalidade: "assistência", "ordem", "denegação", "intracepção", "desempenho", "exibição", "heterossexualidade”, "afago", "mudança", "persistência", "agressão", "deferência", "autonomia", "dominância" e "afiliação". Os 20 itens restantes são concernentes a duas subescalas complementares, uma de desejabilidade social e outra de validade (Pasquali, Azevedo, \& Ghesti, 1997).

A subescala de validade permite verificar se o sujeito respondeu atentamente ao IFP, ao passo que a subescala de desejabilidade social indica se ele tentou se apresentar conforme os desejos de outras pessoas. Tais subescalas, portanto, contribuem para a obtenção de resultados mais precisos do que aqueles decorrentes da aplicação de testes psicológicos semelhantes. Vale destacar também que o IFP é de fácil compreensão, em que pese o fato do sujeito responder aos itens que o constituem escolhendo uma única resposta dentre as opções de uma ampla escala do tipo Likert composta de sete pontos, que variam progressivamente de "nada característico" a "totalmente característico".

Mas a inclusão do IFP no protocolo ora proposto se justifica, para além de suas características técnicas, levando-se em consideração que, a partir da revisão da literatura realizada para os fins do presente estudo, constatou-se que existem evidências empíricas acerca de sua proficuidade no campo da Psico-oncologia. Afinal, o estudo de Peres e Santos (2006) aponta que o teste psicológico em questão se revelou eficiente diante da necessidade de delinear as principais características de personalidade de pacientes oncológicos para o direcionamento da assistência que lhes deve ser oferecida pelo psicólogo hospitalar.

O QSG, por sua vez, é um teste psicológico de rastreamento voltado à avaliação da presença ou ausência de sintomas clínicos não psicóticos típicos de transtornos mentais menores. Investigar a ocorrência de tais transtornos em pacientes oncológicos é relevante posto que, conforme Graner et al. (2008), é elevada a incidência de quadros ansiosos e depressivos nessa população. Tomando como base suas características técnicas, o QSG pode ser considerado o teste psicológico mais indicado para essa finalidade. Contudo, a revisão da literatura realizada para os fins do presente estudo não levou à localização de nenhuma pesquisa nacional reportando o emprego do QSG junto a pacientes oncológicos. 0 estudo de Bertan e Castro (2010), deve-se destacar, envolveu o uso de uma versão abreviada do QSG em uma amostra de adultos acometidos por diversos tipos de câncer. Tal versão abreviada foi preterida no protocolo ora apresentado por não subsidiar uma avaliação tão aprofundada quanto aquela que é possível com a aplicação do QSG.

Afinal, o QSG, em sua versão original, constitui-se de 60 itens que expressam diversos índices comportamentais. As alternativas de respostas são apresentadas em uma escala Likert de quatro pontos, variando de "não, absolutamente" a "muito mais do que de costume". Já os resultados oriundos de sua aplicação são organizados em cinco fatores específicos: "stress psíquico", "desejo de morte", "desconfiança no próprio desempenho", "distúrbios do sono" e "distúrbios psicossomáticos". 0 primeiro destaca as experiências de tensão, irritação, cansaço e sobrecarga que tornam a vida uma luta constante. 0 segundo evidencia a concepção da própria existência como inútil e sem sentido. 0 terceiro expressa a sensação de ser incapaz de desempenhar tarefas diárias de forma satisfatória. 0 quarto se refere a problemas relacionados com o sono, como sua nomenclatura indica. 0 quinto fator, por fim, contempla problemas de ordem orgânica (Pasquali et al., 1996).

Cada um desses fatores é constituído por um conjunto específico de itens. Além disso, há um fator geral, chamado "severidade da ausência de saúde mental", resultante da soma da pontuação de cada um dos 60 itens do instrumento. A presença de sintomas desviantes é verificada quando da ocorrência de escores percentílicos - quer sejam eles referentes ao fator geral ou aos fatores específicos - que se situam acima do ponto de corte estabelecido para a população brasileira pelos responsáveis pela versão nacional do QSG. Entretanto, deve-se esclarecer que a avaliação da presença ou ausência de transtornos mentais menores é feita, conforme Pasquali, Gouveia, 
Andriola, Miranda, e Ramos (1996), a partir da referência aos escores percentílicos do fator geral.

Já o ISSL é um teste psicológico voltado à avaliação dos níveis de estresse e à identificação tanto da natureza sintomatológica quanto da fase de estresse em que se encontra o indivíduo. Conforme Lipp (2000), seus itens são de fácil compreensão e se dividem em três partes. A parte 1 é composta por 15 itens e avalia os sintomas verificados nas últimas 24 horas. A parte 2 possui 15 itens e focaliza a sintomatologia da última semana. A parte 3, por fim, é constituída por 23 itens e se refere aos sintomas ocorridos no último mês. Vale destacar que os itens do ISSL se organizam em um checklist, de modo que o sujeito simplesmente deve assinalar os sintomas que percebeu em si mesmo em cada um dos períodos mencionados. Por essa razão, a aplicação do referido teste psicológico é bastante rápida.

Segundo a definição que fundamenta o ISSL, o estresse pode ser compreendido essencialmente como um conjunto de reações físicas e psicológicas a eventos interpretados, a partir de processos cognitivos individuais, como ameaçadores (Lipp \& Guevara, 1994). Tais eventos tendem a mobilizar diferentes estratégias de enfrentamento, cuja eficiência em termos adaptativos pode ser comprometida se o nível de estresse experimentado pelo sujeito for elevado a ponto de exaurir suas forças. Na medida em que impõem uma nova condição de vida, marcadamente negativa, o diagnóstico e o tratamento do câncer - conforme Levin e Kissane (2006) demonstram em uma ampla revisão da literatura internacional - se destacam como eventos estressores, diante dos quais muitos pacientes oncológicos encontram dificuldades para apresentar respostas adaptativas devido aos elevados níveis de estresse que os acometem.

Torna-se patente, portanto, a necessidade de rastrear a presença de sintomas de estresse nessa população. E o ISSL pode ser considerado o teste psicológico mais adequado para tanto, o que referenda sua inclusão no protocolo ora proposto. Afinal, a revisão da literatura realizada para os fins do presente estudo atestou que, em uma série de estudos nacionais desenvolvidos recentemente no campo da Psico-oncologia (Alves \& Fernandes, 2010; Pelegrini, Cerqueira, \& Peres, 2008; Silva, 2005), o ISSL se mostrou capaz de viabilizar uma avaliação ágil e segura do estresse do sujeito a partir da diferenciação tanto de sintomas dotados ou não de significância clínica quanto de fases que designam a evolução desses sintomas ao longo do tempo.

0 IPSF, por fim, é um teste psicológico voltado à avaliação das relações familiares tais como percebidas pelo indivíduo. Composto por 42 itens, o IPSF se divide em três fatores: "afetivo-consistente", "adaptação familiar" e "autonomia". O primeiro fator, que abrange 21 itens, avalia, essencialmente, a expressão da afetividade, a consistência dos comportamentos e as habilidades na resolução de problemas entre os membros da família. 0 segundo fator investiga, por meio de 13 itens, sentimentos negativos em relação à família, sendo, por essa razão, pontuado de maneira invertida. Já o terceiro fator é constituído pelos oito itens restantes e explora a existência de relações tanto de liberdade quanto de privacidade no âmbito da família (Baptista, 2008).

Os itens do IPSF são de fácil compreensão e se referem a situações familiares diversificadas. As alternativas de respostas são apresentadas em uma escala Likert de três pontos, variando de "nunca" a "quase sempre". Quanto maior a pontuação obtida pelo sujeito, mais adequado pode ser considerado o suporte familiar percebido por ele. Vale destacar também que, apesar de ser o mais recente dos três testes psicológicos do protocolo ora proposto, o IPSF vem sendo adotado, nos últimos anos, em uma série de estudos nacionais. Em parte, esse fato pode ser compreendido em função da carência de testes psicológicos voltados à avaliação familiar. Contudo, tais estudos - a exemplo daqueles realizados por Aquino (2007) e Baptista, Teodoro, Cunha, Santana, e Carneiro (2009) - têm fornecido evidências importantes acerca da proficuidade do IPSF.

A revisão da literatura realizada para os fins do presente estudo, porém, não subsidiou a localização de nenhum estudo nacional no qual o IPSF tenha sido aplicado em pacientes oncológicos. Em que pese esse fato, a avaliação do suporte familiar nessa população pode trazer elementos de grande relevância para o trabalho do psicólogo hospitalar. Ocorre que o apoio dos familiares, quando insuficiente, se afigura como um importante preditor de sintomatologia depressiva, prejuízos no autocuidado e problemas de ajustamento à doença e ao tratamento para os pacientes, comprometendo, assim, a qualidade de vida dos mesmos (Leeuw et al., 2000; Santana, Zanin \& Maniglia, 2008). Portanto, pacientes que apresentam uma percepção negativa do suporte familiar devem receber atenção especial. 
À guisa de conclusão, vale destacar que, como se vê na Figura 1, a aplicação dos testes psicológicos que constituem o protocolo ora proposto poderá subsidiar a definição das condutas a serem adotadas pelo psicólogo hospitalar na assistência ao paciente oncológico. Romano (1999) propõe que tais condutas são basicamente de três tipos: psicopedagógicas, psicoprofiláticas e psicoterapêuticas. As primeiras envolvem, em linhas gerais, a realização de orientação familiar e o fornecimento de informações sobre a doença e tratamento. As segundas consistem na identificação de fontes secundárias de estresse, no fomento à esperança com base na realidade e na intermediação da comunicação com a equipe de saúde. As terceiras, por fim, se caracterizam pelo encorajamento das expressões de emoções, pelo aconselhamento e pelo fornecimento de suporte emocional.

\section{Considerações finais}

Como já mencionado, os protocolos de avaliação psicológica se destacam como um recurso potencialmente proveitoso para o psicólogo hospitalar, na medida em que podem auxiliar na obtenção dos elementos necessários para o planejamento e o desenvolvimento de intervenções. Além disso, são capazes de contribuir para um maior reconhecimento de seu trabalho junto aos demais profissionais de saúde, tendo em vista que viabiliza a definição criteriosa de parâmetros para a proposição de condutas, o que é essencial, sobretudo nos casos em que existem opiniões divergentes no âmbito da equipe multidisciplinar acerca do tratamento a ser oferecido aos pacientes.

Não obstante, o protocolo proposto por Azevêdo (2010) para utilização junto a crianças queimadas hospitalizadas foi o único localizado a partir da revisão da literatura nacional realizada para os fins do presente estudo. 0 protocolo ora proposto, portanto, tem o mérito de fornecer subsídios iniciais para o preenchimento de uma importante lacuna na produção científica brasileira em Psicologia Hospitalar e, mais especificamente, em Psico-oncologia. Uma maior articulação entre a pesquisa e a prática se afigura como uma condição indispensável, como bem observou Costa (2001), para a superação do principal desafio que se apresenta à Psico-oncologia: a estruturação de um modelo de atuação que, independentemente da abordagem teórico-filosófica adotada pelo psicólogo, se revele eficiente no sentido de promover a qualidade de vida dos pacientes oncológicos.

Experiências preliminares realizadas pelos autores do presente estudo revelaram que o protocolo ora proposto possibilita a obtenção de informações de grande relevância para a prática do psicólogo hospitalar junto ao paciente oncológico. Porém, pesquisas posteriores são necessárias para uma avaliação mais detalhada de sua resolutividade. Além disso, é preciso salientar que o protocolo ora proposto pode ser adaptado de diferentes maneiras. Caso o psicólogo hospitalar disponha de um tempo mais restrito, obviamente é possível privilegiar alguns dos testes psicológicos em detrimento de outros. Por outro lado, diante de uma eventual necessidade de maior aprofundamento, o protocolo ora proposto pode ser complementado a partir do recurso a outros testes psicológicos, tais como os testes projetivos, por exemplo.

Por fim, faz-se necessário esclarecer que, como destacam Capitão et al. (2005), para que os protocolos de avaliação possam, de fato, contribuir para o planejamento e o desenvolvimento de intervenções eficientes, cabe ao psicólogo hospitalar utilizá-los em conformidade com as condições de cada paciente. Afinal, deve-se levar em conta diversos fatores médicos, sociais, culturais e ambientais que podem influenciar os resultados decorrentes da avaliação. Quando isso não ocorre, os protocolos acabam limitando a compreensão, por parte do psicólogo hospitalar, da subjetividade de seus pacientes, o que o levará a uma prática mecanicista e desumanizada.

\section{Referências}

Almeida, S. R. (1995). Alcances e limites do uso de testes psicológicos no hospital. In M. F. P. Oliveira \& S. M. C. Ismael (Org.). Rumos da psicologia hospitalar em cardiologia (pp. 147-152). Campinas: Papirus.

Alves, C. A., \& Fernandes, A. F. C. (2010). Estresse e estratégias de enfrentamento/coping diante do câncer de mama: Nota prévia. Online Brazilian Journal of Nursing, 9(1). Recuperado em 3 de abril, 2014, de http://www.objnursing.uff.br/index.php/nursing/ article/view/j.1676-4285.2010.2861/635 
Angerami-Camon, V. A.; Chiattone, H. B. C. (2003). E a psicologia entrou no hospital. São Paulo: Pioneira.

Aquino, R. R. (2007). Inventário de Percepção de Suporte Familiar (IPSF) e Escala de Vulnerabilidade ao Estresse no Trabalho (EVENT): Evidência de validade. Dissertação de Mestrado, Universidade São Francisco, Itatiba.

Azevêdo, A. V. S. (2010). Construção do protocolo de avaliação de psicológica hospitalar para criança queimada. Avaliação Psicológica, 9(1), 99-109.

Baptista, M. N. (2005). Desenvolvimento do Inventário de Percepção de Suporte Familiar (IPSF): Estudos psicométricos preliminares. Psico-USF, 10(1), 11-19.

Baptista, M. N. (2008). Inventário de Percepção de Suporte Familiar. São Paulo: Vetor.

Baptista, M. N., Teodoro, M. L. M., Cunha, R. V., Santana, P. R., \& Carneiro, A. M. (2009). Evidência de validade entre o Inventário de Percepção de Suporte Familiar (IPSF) e Familiograma (FG). Psicologia: Reflexão e Crítica, 22(3), 466-473.

Barbosa, L. N. F., Santos, D. A., Amaral, M. X. F., Gonçalves, A. J., \& Bruscato, W. L. (2004). Repercussões psicossociais em pacientes submetidos à laringectomia total por câncer de laringe: Um estudo clínico-qualitativo. Revista da SBPH, 7(1), 45-58.

Bertan, F. C., \& Castro, E. K. (2010). Qualidade de vida, indicadores de ansiedade e depressão e satisfação sexual em pacientes adultos com câncer. Salud y Sociedad, 1(2), 76-88.

Bruscato, W. L., \& Benedetti, C. (2004). Produção de conhecimento em psicologia hospitalar. In W. L. Bruscato, C. Benedetti \& S. R. A. Lopes (Org.). A prática da psicologia hospitalar na Santa Casa de São Paulo: Novas páginas em uma antiga história (pp. 213-236). São Paulo: Casa do Psicólogo.

Carvalho, M. M. M. J. (2002). Psico-oncologia: História, características e desafios. Psicologia USP, 13(1), 151-166.

Capitão, C. G., Scortegagna, S. A., \& Baptista, M. N. (2005). A importância da avaliação psicológica na saúde. Avaliação Psicológica, 4(1), 75-82.

Chiattone, H. B. C. (1996). Uma vida para o câncer. In V. A. Angerami-Camon, H. B. C. Chiattone \& E. A. Nicoletti (Org.). O doente, a psicologia e o hospital (pp. 73-110). São Paulo: Pioneira.
Resolução CFP n. 02/2003. (2003). Define e regulamenta o uso, a elaboração e a comercialização de testes psicológicos e revoga a Resolução CFP nº 025/2001. Brasília. Recuperado em 3 de abril, 2014, de http:// www.pol.org.br/legislacao/pdf/resolucao2003_2.pdf

Costa, A. L., Jr. (2001). O desenvolvimento da Psicooncologia: Implicações para a pesquisa e intervenção profissional em saúde. Psicologia: Ciência e Profissão, 21(2), 36-43.

Dalgalarrondo, P. (2000). Psicopatologia e semiologia dos transtornos mentais. Porto Alegre: Artmed.

Graner, K. M., Cezar, L. T. S., \& Teng, C. T. (2008). Transtornos do humor em Psico-oncologia. In V. A. Carvalho et al. (Org.). Temas em Psico-oncologia (pp.243-256). São Paulo: Summus.

Hogan, T. P. (2006). Introdução à prática de testes psicológicos. Rio de Janeiro: LTC.

Holland, J. C. (2002). History of psycho-oncology: Overcoming attitudinal and conceptual barriers. Psychosomatic Medicine, 64(2), 206-221.

Leeuw, J. R. J., Graeff, A. D., Ros, W. J. G., Blijham, G. H., Hordijk, G. J., \& Winnubst, J. A. M. (2000). Prediction of depressive symptomatology after treatment of head and neck cancer: The influence of pre-treatment physical and depressive symptoms, coping, and social support. Head \& Neck, 22(8), 799-807.

Levin, T., \& Kissane, D. W. (2006). Psychooncology: The state of its development in 2006. European Journal of Psychiatry, 20(3), 189-197.

Lipp, M. E. N. (2000). Manual do Inventário de Sintomas de Stress para Adultos de Lipp (ISSL). São Paulo: Casa do Psicólogo.

Lipp, M. E. N., \& Guevara, A. J. H. (1994). Validação empírica do Inventário de Sintomas de Stress (ISS). Estudos de Psicologia, 3(11),43-49.

Lopes, S. R. A., \& Amorim, S. F. (2004). Avaliação psicológica no hospital geral. In W. L. Bruscato, C. Benedetti, \& S. R. A. Lopes (Org.). A prática da psicologia hospitalar na Santa Casa de São Paulo: Novas páginas em uma antiga história (pp.53-68). São Paulo: Casa do Psicólogo.

Pasquali, L., Azevedo, M. M., \& Ghesti, I. (1997). Inventário Fatorial de Personalidade: Manual técnico e de aplicação. São Paulo: Casa do Psicólogo. 
Pasquali, L., Gouveia, V. V., Andriola, W. B., Miranda, F. J., \& Ramos, A. L. M. (1996). Questionário de Saúde Geral de Goldberg: Manual técnico. São Paulo: Casa do Psicólogo.

Pelegrini, L. G., Cerqueira, J. A., \& Peres, R. S. (2008). Indicadores de qualidade de vida e sintomas de ansiedade, depressão e estresse em mulheres mastectomizadas no período de reabilitação. Revista $d a$ Sociedade de Psicologia do Triângulo Mineiro, 12(2), 168-176.

Peres, R. S., \& Santos, M. A. (2006). Contribuições do Inventário Fatorial de Personalidade (IFP) para a avaliação psicológica de pacientes onco-hematológicos com indicação para o transplante de medula óssea. Psicologia em Revista, 12(19), 22-33.

Ribeiro, V. (2005). Diferenças entre o atendimento psicológico em meio hospitalar e em consultório. Revista Virtual de Psicologia Hospitalar e da Saúde, 1(1), 14-16.
Romano, B. W. (1999). Princípios para a prática da psicologia clínica em hospitais. São Paulo: Casa do Psicólogo.

Santana, J. J. R. A., Zanin, C. R., \& Maniglia, J. V. (2008). Pacientes com câncer: Enfrentamento, rede social e apoio social. Paidéia, 18(40), 371-384.

Silva, G. (2005). Processo de enfrentamento no período pós-tratamento do câncer de mama. Dissertação de Mestrado, Universidade de São Paulo, Ribeirão Preto.

Simonetti, A. (2004). Manual de psicologia hospitalar: $O$ mapa da doença. São Paulo: Casa do Psicólogo.

Veit, M. T. \& Carvalho, V. (2008). Psico-oncologia: conceituação, definições, abrangência de campo. In: V. A. Carvalho et al. (Org.). Temas em Psico-oncologia (pp. 15-20). São Paulo: Summus. 